\title{
DESENVOLVIMENTO DE UM TENSOATIVO A BASE DE ÓLEO DE BABAÇU PARA FORMULAÇÃO DE MICROEMULSIONADOS USADO PARA QUEBRA DE EMULSÕES DO TIPO ÁGUA EM ÓLEO
}

\section{DEVELOPMENT OF A BABAÇU OIL BASED TENSIONER FOR FORMULATION OF MICROEMULSIONED SYSTEMS USED FOR BREAKING OF EMULSIONS OF THE OIL WATER TYPE}

\author{
M. F. O. SANTOS ${ }^{1, *}$, V. A. S. JESUS ${ }^{1}$, G. F. SILVA ${ }^{1}$, M. S. SILVA ${ }^{1}$, J.P. L. SANTOS ${ }^{1}$. \\ ${ }^{1}$ Universidade Federal de Sergipe, Núcleo de Graduação em Engenharia de Petróleo, São Cristóvão, Sergipe, Brasil \\ Universidade Federal de Sergipe, Núcleo de Graduação em Engenharia de Petróleo, São Cristóvão, Sergipe, Brasil. Phone: +55 79 31946593. e-mail addressl: \\ mfernanda2202@hotmail.com (M.F.OLIVEIRA).
}

\begin{tabular}{|c|}
\hline A R T I C LE I NFO \\
\hline $\begin{array}{l}\text { Article history: } \\
\text { Received 2018-10-12 } \\
\text { Accepted 2018-12-22 } \\
\text { Available online 2019-04-25 }\end{array}$ \\
\hline $\begin{array}{l}\text { palavras-chave } \\
\text { Emulsões } \\
\text { Microemulsões } \\
\text { Quebra de emulsão } \\
\text { Tensoativo }\end{array}$ \\
\hline $\begin{array}{l}\text { keywords} \\
\text { Emulsions } \\
\text { Microemulsions } \\
\text { Emulsion break } \\
\text { Surfactant }\end{array}$ \\
\hline
\end{tabular}

\begin{abstract}
A B S T R A C T
During the oil production process, the formation of emulsions initiate a number of problems that harm from production to refining. The main problems caused by the presence of water are relacioned to the corrosion process, increase in the custs of pumping and flow, inorganic incrustacions in equipaments, among others. Trying to solve these problems, the industry has aroused interest in the application of microemulsion systems. The microemulsions are mixtures with low interfacial tension, high stability and with solubility capacity of both aqueous and oily substances being feasible in the break of emulsions. In this work, developed a surfactant the base of saponified babassu oil (OBS) was used to construct pseudo-ternary phase diagram for determination Winsor IV regions of two microemulsions systems: $C / T=10$ ( $\mathrm{n}$ butanol/OBS) and babassu oil in oily phase (system 1); $C / T=10$ (isoamylic/OBS) and toluene in oily phase (siystem 2). Besides that, the surfactant L19 was selected for a comparative analysis of the emulsion brake efficiency in relation to the others systems. The spots were applied to an oil characterized by BSW of 24\%, API of 23.57 and specific mass of $0.912 \mathrm{~g} / \mathrm{ml}$, and the reading of the water volume separated was performed by the break test. As a resulted of experiment, it was obtained wich the system 2 presented an greater region Winsor IV, but the spot of the systm 1 triggered a greater efficiency on the brake of emulsion in 21,68\% in relation to the others.

R E S U M O / R E S U M E N
\end{abstract}

Durante o processo de produção de petróleo, a formação de emulsões desencadeia um conjunto de problemas que prejudica da produção até o refino. Os problemas básicos devido a existência de água estão associados aos processos de corrosão, aumento dos custos de bombeamento e escoamento, incrustação inorgânica nos equipamentos, entre outros. Para tentar solucionar este problema, a indústria tem despertado interesse na aplicação do sistema microemulssionados. As microemulsões são misturas com tensão interfacial baixa, alta estabilidade e com capacidade de solubilização tanto de substâncias aquosas quanto de oleosas sendo exequível na quebra de emulsões. Neste trabalho, foi desenvolvido um tensoativo a base de óleo de babaçu saponificado (OBS) utilizado para construção dos diagramas de fases pseudoternários para determinação da região de Winsor IV de dois sistemas microemulssionados: C/T = 10 (nbutanol/ OBS) e óleo de babaçu na fase oleosa (sistema 1); $\mathrm{C} / \mathrm{T}=10$ (isoamílico/OBS) e tolueno na fase oleosa (sistema 2). Além disso foi selecionado o desemulsificante L19 para uma análise comparativa da eficiência na quebra da emulsão em relação aos demais sistemas. Os pontos foram aplicados a um petróleo caracterizado por BSW de 24\%, ${ }^{\circ} \mathrm{API}$, de 23,57 e massa específica de $0,912 \mathrm{~g} / \mathrm{ml}$ e observou-se leitura do volume de água separada pelo teste de quebra. Como resultado do experimento, obteve-se que o sistema 2 apresentou uma maior região de Winsor IV, entretanto o ponto do sistema 1 desencadeou uma maior eficiência na quebra de emulsão de $21,68 \%$ comparado os outros. 


\section{INTRODUÇÃO}

$\mathrm{Na}$ indústria petrolífera ao longo do processo de produção, pode surgir água oriunda de aquíferos ou proveniente dos mecanismos de recuperação secundária por injeção de água [ARAÚJO, 2004]. A presença da água pode estar na forma de água livre permanecendo instável e, consequentemente, permitindo a separação por decantação ou emulsionada no petróleo de forma estável permitindo realizar mecanismos de quebra de emulsão, como por exemplo, através da eletrostática ou através de sistemas microemulsionados. Além disso, a água que forma as emulsões pode conter sais, causadores de oxidação nos dutos e de incrustações que obstruem a passagem do petróleo no duto [ARAÚJO, 2004].

A emulsão é formada quando um líquido se dispersa em outro em forma de gotículas, sendo eles imiscíveis entre si [SCHRAMM, 1992]. Isso só ocorre na presença de um agente emulsificante e da energia cisalhante imposta ao fluido durante a produção. Os agentes emulsificantes possuem propriedades hidrofílicas e lipofílicas respectivamente e atua na interface entre líquido-líquido reduzindo a tensão interfacial entre os mesmos, que dificulta a coalescência das gotículas. Normalmente, a formação dessas emulsões em campos de petróleo é considerada indesejável, pois pode causar sérios problemas nas operações de transporte, armazenamento e refino.

Os sistemas microemulsionados têm motivado estudos por causa da sua ampla capacidade e aplicações industriais. As microemulsões são misturas com tensão interfacial baixa, alta estabilidade e com capacidade de solubilização tanto de substâncias aquosas quanto de oleosas sendo exequível na quebra de emulsões. [SANTOS, et. al. 2007]. A formulação do sistema microemulsionado, na maioria das vezes, envolve a combinação de três a cinco componentes: óleo, água, tensoativo, cotensoativo e eletrólito [ABOOFAZELI et al., 1993].

Este trabalho tem a finalidade realizar um estudo sobre a influência da variação dos componentes do sistema sobre a região microemulsionada e testar a eficiência da quebra para estes diferentes sistemas.

\section{MATERIAIS E METODOLOGIA}

O petróleo utilizado para a caracterização foi o da Bacia Sergipe-Alagoas. Inicialmente foi feita a determinação do BSW (Basic sedmentes and water) através da NBR 14647:2010. Determinou-se a massa específica utilizando-se o método do picnômetro a temperatura ambiente [ANDRADE et al., 2017]. E para classificar o óleo utilizou-se o ${ }^{\circ} \mathrm{API}$ (American Petroleum Institute) [ROSA et. all., 2011]. Para a determinação da viscosidade foi utilizado um Reômetro da Lamy Technology modelo RM300, acoplado a um banho termostatizado da Tecnal. As curvas reológicas do reômetro foram obtidas através do software Rhematic-P, alterando a temperatura durante cada ensaio para uma quantidade de $20 \mathrm{ml}$ de óleo.

$\mathrm{Na}$ formulação do tensoativo a base de óleo de babaçu utilizou-se o processo de saponificação através da metodologia presente em [ANDRADE, 2017]. O tensoativo obtido foi denominado óleo de Babaçu Saponificado (OBS). O procedimento utilizado para obtenção dos diagramas pseudoternários e para região de microemulsão, baseia-se na titulação volumétrica, onde em um béquer mistura-se a fase oleosa e a razão $\mathrm{C} / \mathrm{T}$ em proporções conhecidas determinada pela razão $\mathrm{C} / \mathrm{T}$, e em seguida titula-se a mistura, mantida à temperatura ambiente, com a fase aquosa até o ponto de viragem [RAMOS, 1996 apud SANTOS, 2013]. Este ponto é caracterizado pela mudança de aspecto do sistema, que sob agitação, passa de límpido para turvo, ou vice-versa, indicando, respectivamente, a saída e a entrada da região de Winsor IV (microemulsão) [ARAÚJO, 2004]. Nos experimentos a massa total da mistura de $\mathrm{C} / \mathrm{T}$ e fase oleosa foi fixado em dois gramas, partindo-se do ponto inicial com $0 \%$ de óleo e $100 \% \mathrm{C} / \mathrm{T}$ e fazendo incrementos na concentração de óleo de 10\% [SOUZA, 2015]. Assim, podem-se obter vários pontos da curva que separa as regiões de formação e não formação de microemulsão, para sucessiva construção dos diagramas.

Foram testados dois tipos de cotensoativo (n-butanol e álcool isoamílico), duas fases oleosas (óleo de babaçu e Tolueno) e três diferentes razões $\mathrm{C} / \mathrm{T}$ (1, 5 e 10) visando determinar as condições que se obtém a maior região de microemulsão.

$\mathrm{Na}$ seleção do diagrama a ser aplicado na quebra da emulsão levou-se em consideração a maior região de microemulsão de Winsor IV [SOUZA, 2015]. E o segundo critério foi o que apresenta uma área de microemulsão rica em óleo e que fosse de fácil obtenção [ARAÚJO, 2004].

$\mathrm{Na}$ seleção dos pontos a serem analisados dentro das maiores regiões de microemulsão utilizou-se a rede de Scheffé. Utilizou-se a suposição de um modelo linear de grau unitário (Equação 1) e se baseando nisso construiu-se um triângulo equilátero dentro da região a ser estudada, onde cada vértice representa um ponto a ser estudado e o ponto central, serve para a validação do sistema (Figura1) [ARAÚJO, 2004].

$$
Y=A . X_{1}+B . X_{2}+C . X_{3}
$$

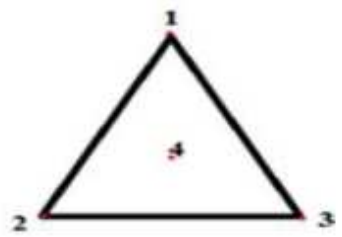

Figura 1 - Rede de Scheffé (Grau 1). [SOUZA, 2015]

A validação ocorre quando o valor teórico for muito próximo ao valor encontrado experimentalmente nos ensaios de quebra de emulsão. Caso isso não ocorra, utiliza-se a hipótese do modelo linear de grau dois, neste amplia-se a quantidade de pontos a serem estudados e a validação se dá com os pontos centrais dos novos triângulos equiláteros. Essa interação é feita até a validação do sistema [SOUZA, 2015]. Para a quebra da emulsão foi utilizado a metodologia presente em [ANDRADE et al., 2017]. A eficiência de separação da água foi dada pela Equação (2), onde: $\mathrm{BSW}_{\mathrm{i}}$ é o teor de água e sedimentos inicial e $\mathrm{BSW}_{\mathrm{f}}$ é o teor de água e sedimentos final do petróleo.

$$
\text { Eficiência }(\%)=\frac{B S W_{i}-B S W_{f}}{B S W_{i}} \cdot 100
$$




\section{RESULTADOS E DISCUSSÕES}

Através da norma ABNT NBR 14647:2010 determinouse que o teor de água e sedimentos presentes no petróleo foi de $24 \%$. Os resultados referentes a determinação da massa específica do petróleo foi de $0,912 \mathrm{~g} / \mathrm{ml}$ e do ${ }^{\circ} \mathrm{API}$, foi de 23,57 classificando o óleo como de grau médio [SZKLO, 2005]. Conforme os dados obtidos, observou-se que massa específica do petróleo é menor que a da água [ANDRADE et al., 2017], logo a separação das fases ocorrerá de modo que a água seja a fase inferior e o óleo a fase superior. Além disso, devido a diferença de densidades das fases ser pequena é mais difícil que ocorra a separação gravitacional, logo pode-se afirmar que a quebra da emulsão A/O apresentará um grande grau de dificuldade. A viscosidade do petróleo é igual a $(0,361 \pm 0,005)$ Pa.s, este valor apesar de ser alto é condizente para óleos classificação como médios.

Usando o método descrito no item 2 determinou-se as regiões de Winsor IV. Trabalhou-se com o sistema microemulsionado de Óleo de Babaçu saponificado (OBS) como tensoativo, o n-butanol como cotensoativo, a água destilada como fase aquosa e o óleo de babaçu saponificado (OBS) como fase oleosa. E então, variou-se a razão cotensoativo/tensoativo (C/T) entre 1, 5 e 10, obtendo-se os diagramas, conforme ilustra as Figuras de 2 e 3 Além disso, foi realizado outro teste modificando o cotensoativo para isoamílico e a fase oleosa para tolueno. Entretanto, para este último optouse por fazer apenas o diagrama do $C / T=10$, pois é o que apresenta maior região de Winsor IV.

a)

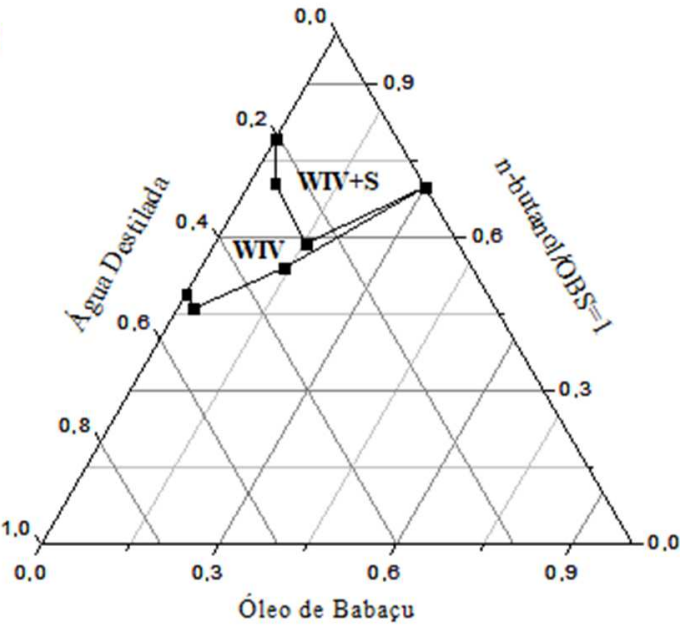

b)

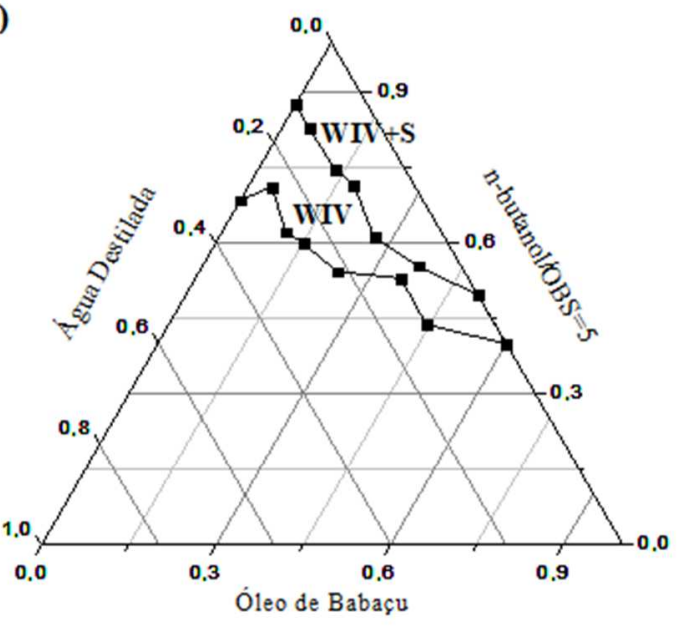

Figura 2 - Diagramas de fases: $C=n$-butanol a) $C / T=1$; b) $\mathrm{C} / \mathrm{T}=5$. Próprio autor.
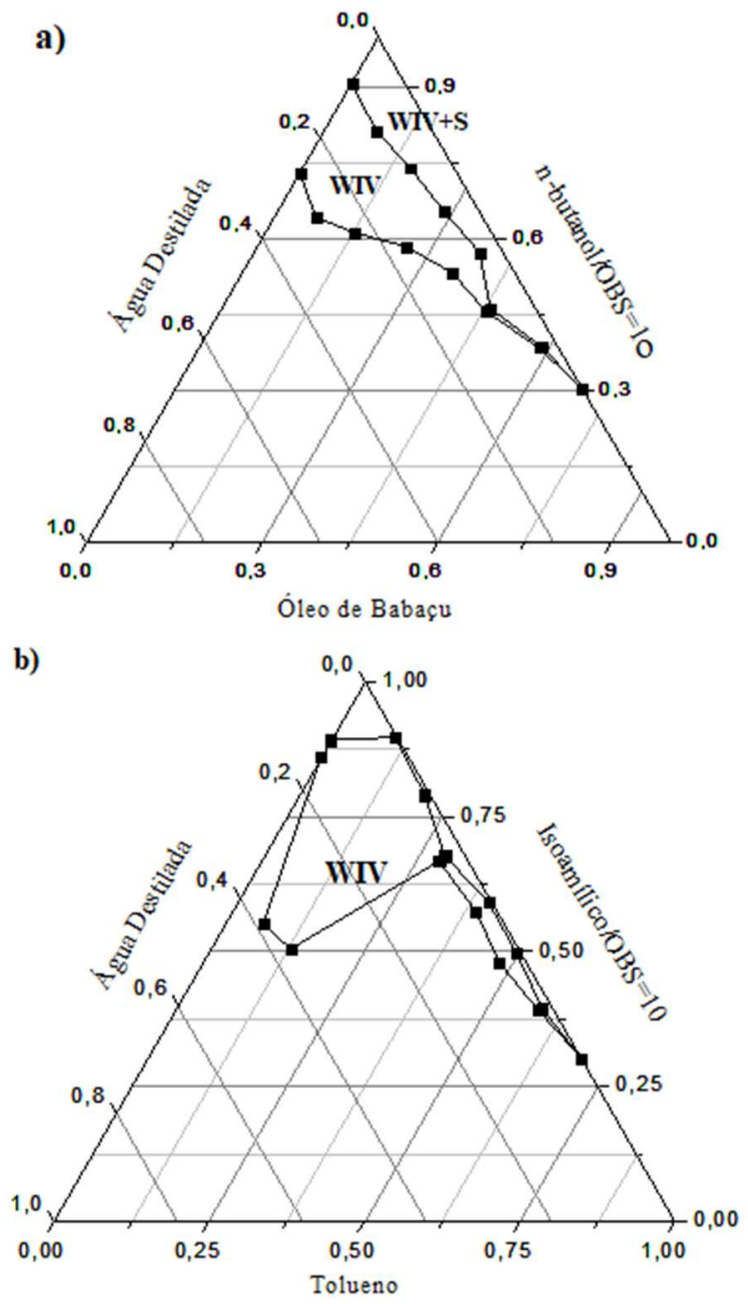

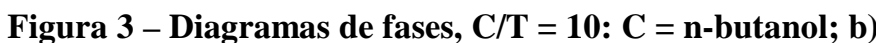
$\mathrm{C}=$ Isoamílico. Próprio autor.

$\mathrm{O}$ diagrama $\mathrm{C} / \mathrm{T}=1$ possui menor quantidade de cotensoativo e o tensoativo possui caráter hidrofílico, então pode-se afirmar que o sistema é do tipo óleo em água, ou seja, a água encontra-se em maior concentração e o óleo é a fase dispersa. No caso dos diagramas $\mathrm{C} / \mathrm{T}=5$ e 10 , onde as quantidade de cotensoativo é maior, notou-se que o tensoativo passou a ter maior afinidade por óleo. Tal fato pode ser explicado, pois quando há a adição de álcoois provoca a diminuição do valor de BHL do tensoativo em questão, e, por consequência, a formação de sistemas microemulsionados do tipo A/O [ANDRADE et al., 2017]. Logo, observou-se a inversão de microemulsão $\mathrm{O} / \mathrm{A}$ para $\mathrm{A} / \mathrm{O}$, em função da alteração da composição.

De acordo com os diagramas obtidos com o uso de OBS (Figura 2 e 3 a) e seguindo o primeiro critério descrito no item 2 pode-se concluir que o $\mathrm{C} / \mathrm{T}=10$ é o que apresenta maior região de WIV (ver Figura 3) e é interessante para aplicação na quebra de emulsão de petróleo. De acordo com a Figura 3 e com o critério, quando a razão $\mathrm{C} / \mathrm{T}$ vai aumentando, a região de microemulsão fica cada vez mais visível, o que se torna interessante do ponto de vista econômico, visto que a parcela onerosa (tensoativo) diminui. Além disso, as Figura 2 e 3 com o aumento da razão $\mathrm{C} / \mathrm{T}$ provoca o aumento da afinidade pelo óleo. Logo, pelo critério dois descrito no item 2 , o C/T $=10$ é o 
mais interessante para aplicação na quebra de emulsões de petróleo. Com os diagramas selecionados (Figuras 4 (a) e (b) foi possível determinar os pontos, dentro das regiões de interesse WIV, para que sejam utilizados na quebra da emulsão e criar a rede Scheffé.
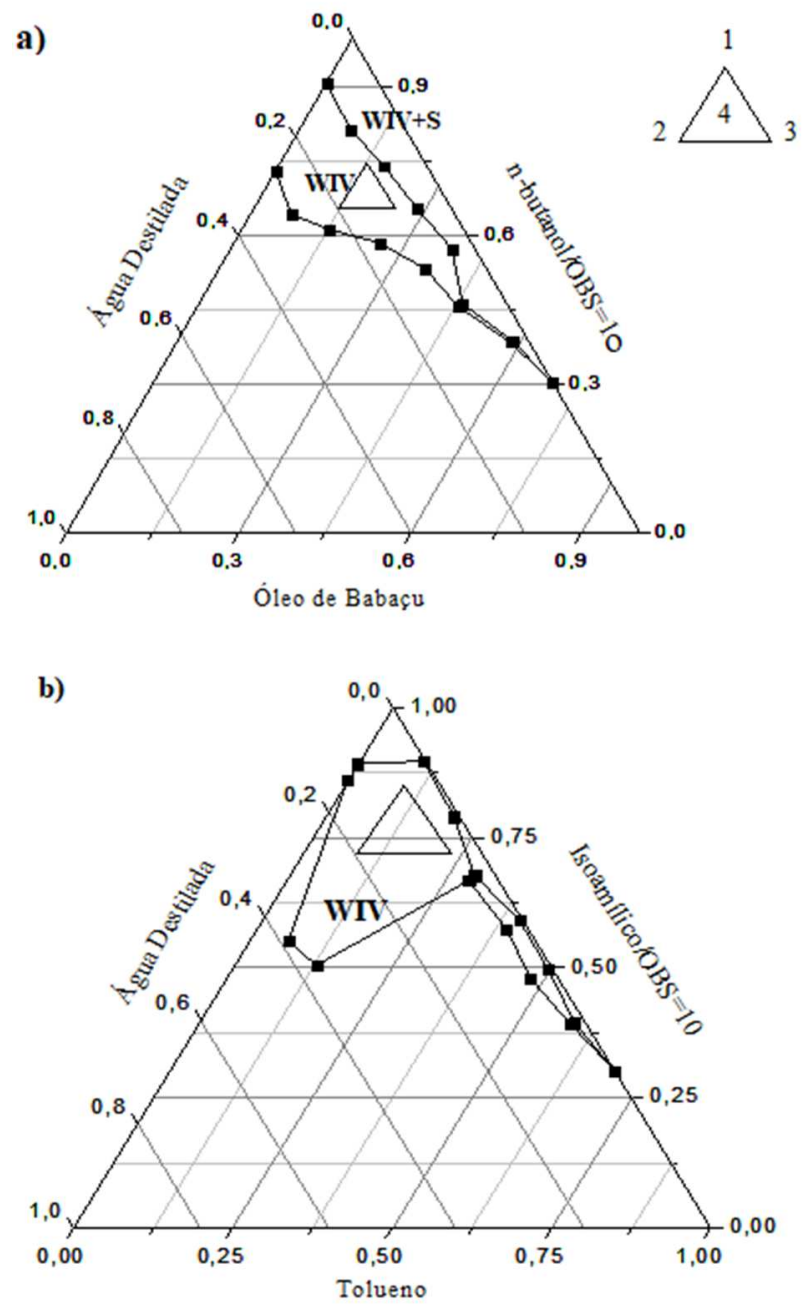

Figura 4 - Rede de Scheffé, C/T=10: a) OBS/n-butanol, b) OBS/Isoamilíco. Próprio autor.

Para verificar a quebra da emulsão, usou-se o método descrito no item 2 e traçou-se as curvas da Figura 9 a e b.

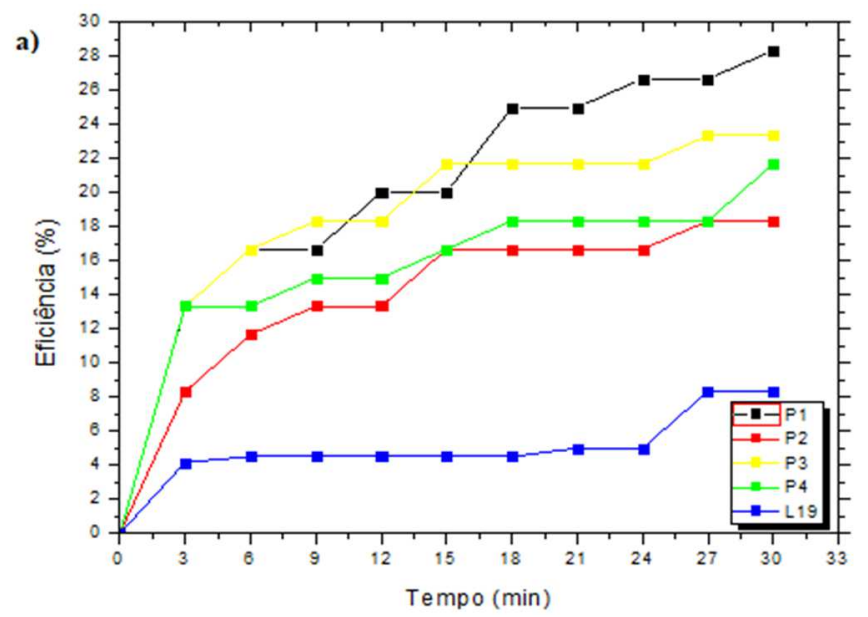

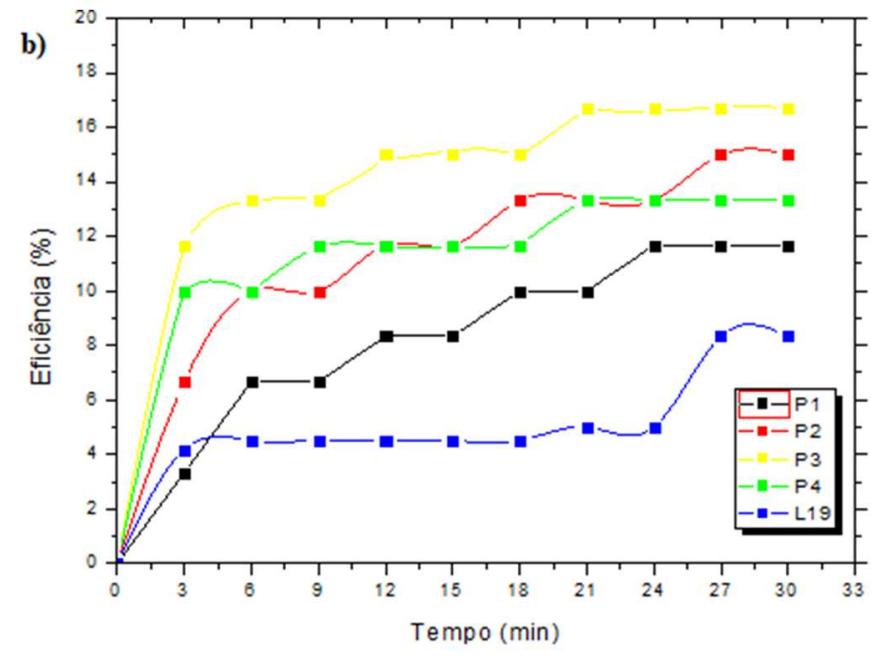

Figura 5 - Eficiência da quebra da emulsão de petróleo versus o tempo para o sistema microemulsionado: a) OBS/n-butanol = 10; b) OBS/Isoamilíco = 10. Próprio autor.

Aplicou-se o modelo linear da rede Scheffé (Equação 1) para iniciar os estudos. Então, obteve-se, para cada microemulsão, um sistema linear de três equações (ver Tabela 1) e três incógnitas, e os coeficientes do sistema $\left(X_{C / T}, X_{O}\right.$ e $\left.X_{A}\right)$ estão apresentados na Tabela 2.

Tabela 1 - Sistema linear para as microemulsões. Próprio autor.

Por fim, para que o modelo fosse validado, se dá com a aplicação no ponto 4 dos coeficientes (A, B e C) que foram

\begin{tabular}{llll} 
OBS/n-butanol = 10 & & Tolueno/Isoamilíco = 10 & \\
$0,75 A+0,15 B+0,10 C=\mathrm{Q}$ & 7 & $0,84 A+0,10 B+0,06 C=\mathrm{Q}$ & 10 \\
$0,65 \cdot A+0,15 B+0,20 C=\mathrm{Q}$ & 8 & $0,70 A+0,10 B+0,20 C=\mathrm{Q}$ & 11 \\
$0,65 A+0,25 B+0,10 C=\mathrm{Q}$ & 9 & $0,70 A+0,24 B+0,06 C=\mathrm{Q}$ & 12 \\
\hline
\end{tabular}
determinados pelo sistema citado anteriormente. Foi admitido um intervalo de erro de 0 a $10 \%$ para a aceitação das respostas da separação de água.

As Equações 3 e 4 representam os coeficientes determinados pela resolução dos sistemas com o OBS/n-butanol $=10$ e OBS/Isoamilíco $=10$, respectivamente:

$$
\begin{aligned}
& 0,458 X_{C / T}-0,0417 X_{O}-0,542 X_{A}=Q \\
& 0,0677 X_{C / T}+0,424 X_{O}+0,305 X_{A}=Q
\end{aligned}
$$

Através da Equação 2 calculou-se a eficiência experimental da quebra de emulsão de petróleo para cada ponto do $\mathrm{C} / \mathrm{T}=10$ (ver Tabela 2).

Tabela 2 - Valores das eficiências experimentais de cada ponto. Próprio autor.

\begin{tabular}{|ccccccccc|}
\hline \multicolumn{3}{|c}{ OBS/n-butanol = 10 $(\boldsymbol{\%})$} & \multicolumn{4}{c|}{ Tolueno/Isoamilíco = 10 (\%) } \\
\hline P1 & P2 & P3 & P4 & P1 & P2 & P3 & P4 \\
\hline 28,33 & 18,33 & 23,33 & 21,68 & 11,67 & 15,00 & 16,67 & 13,33 \\
\hline
\end{tabular}

Resolvendo as Equações 3 e 4 encontrou-se uma eficiência teórica de $23,33 \%$ para o ponto 4 do OBS/n-butanol e uma de $14,49 \%$ para o ponto 4 do Tolueno/Isoamilíco. Quando comparado os valores anteriores com as eficiências experimentais do ponto 4 (ver Tabela 2) encontrou-se um erro 
de 7,63 \% para o OBS/n-butanol e 8,52 \% para o Tolueno/Isoamilíco, indicando que o modelo apresentado é válido para o sistema analisado.

Assim o OBS/n-butanol, $\mathrm{C} / \mathrm{T}=10$ foi o mais eficiente na quebra. Este fato pode ser justificado pelo fato do sistema ser rico em óleo e apresentar maior eficiência para solubilizar as gotículas de água e, assim, provocar a sua separação.

\section{CONCLUSÃO}

Conforme os resultados obtidos, percebe-se que o tensoativo produzido (OBS) apresenta alta polaridade e alta capacidade de atração de moléculas de água. Isso faz com que sejam mais solúveis em água e, portanto, uma ótima opção para utiliza-lo no sistema microemulssionado. Além disso, diante da comparação entre os diagramas com diferentes $\mathrm{C} / \mathrm{T}$ (1, 5 e 10) para $\mathrm{C} / \mathrm{T}=\mathrm{n}$-butanol/OBS, água destilada como fase aquosa $\mathrm{e}$ óleo de babaçu como fase óleo (Sistema 1), foi possível constatar que o aumento da razão $\mathrm{C} / \mathrm{T}$ provoca um aumento na região de microemulsão e isto se torna interessante, pois além de reduzir o consumo de tensoativo facilita na escolha dos pontos que serão utilizados na etapa de quebra de emulsão. Esse resultado justifica a construção de apenas um diagrama com $\mathrm{C} / \mathrm{T}=10$ utilizando $(\mathrm{C} / \mathrm{T}=$ isoamílico/OBS $)$, água destilada como fase aquosa e tolueno como fase óleo (Sistema 2). Posteriormente, foi possível realizar uma análise comparativa entre os diagramas $\mathrm{C} / \mathrm{T}=10$ para os dois sistemas e o sistema 2 obteve uma maior região de microemulsão. Entretanto, ao aplicar separadamente o ponto de microemulsão dos dois sistemas e também o desemulsificante L19 no petróleo caracterizado por BSW de $24 \%$, ${ }^{\circ} \mathrm{API}$, de 23,57 e massa específica de $0,912 \mathrm{~g} / \mathrm{ml}$, obteve-se que o sistema 1 apresenta uma maior quebra de emulsão com uma eficiência de $21,68 \%$. Esse fato mostra que as ME's ricas em óleo e especificadamente com cadeia alquílicas maiores, apresentam alta eficiência no tratamento de emulsão de petróleo, pois favorecem a formação de estrutura diretas, ou seja água em óleo que ao entrarem em contato com a emulsão de petróleo, tendem a solubilizar as gotículas de água e consequentemente provocar uma maior separação de fase. Portanto, pode-se perceber que uma boa capacidade desse sistema 1 para ser usado com agente desemulsificante de petróleo.

\section{R E F E R E N C E S}

ABOOFAZELI R., LAWRENCE MJ. Investigations into the Formation and Characterization of Phospholipid Microemulsions .1. Pseudo-Ternary Phase-Diagrams of Systems Containing Water-Lecithin-Alcohol-Isopropyl Myristate. Int J Pharm. 1993 May 31; 93(1-3):161-75.

ANDRADE. W. A. A, CRUZ G. P., SILVA M. S., SANTOS M. F. O., SILVA G. F., SANTOS J. P. L. Síntese de um tensoativo a base de óleo de moringa oleífera lam. E formulação de sistemas microemulsionados para a quebra de emulsões do tipo água em óleo. In:. IX SEPEQ., São Cristovão, SE, 2017. p.
Foram feitos testes de eficiência de quebra experimental com o desemulsificante comercial L19 para comparar a separação com os demais sistemas microemulsionado. A eficiência experimental para este caso foi de $8,33 \%$. O resultado apresentado mostra uma baixa eficiência de separação das fases tal fato pode ser explicado, pois a eficiência do desemulsificante depende da composição da espécie química, concentração, além de depender das características e tempo do contato entre o óleo e o mesmo [HILÁRIO, 2012].

ARAÚJO, M. M. S. Estudo de Quebra de Emulsões de Petróleo Utilizando Microemulsões e Célula de Desidratação Eletrostática. 2004. Dissertação de Mestrado (Programa de Pós-Graduação em Engenharia Química), Áreas de Concentração: Tecnologia de Tensoativos e Processos de separação - Universidade Federal do Rio Grande do Norte, Natal/RN, Brasil., 2004.

HILÁRIO, L. S. Avaliação de desempenho de desemulsificantes comerciais na separação da água produzida do petróleo. 2012. Trabalho de conclusão de curso - Universidade Federal do Rio Grande do Norte centro de tecnologia, Natal/RN dezembro/2012.

RAMOS, A. C. S. Extração de Tungstênio utilizando microemulsões. 1996. Dissertação de Mestrado (Programa de Pós-Graduação em Engenharia Química) Universidade Federal do Rio Grande do Norte, 1996.

ROSA, A. J.; CARVALHO, R. S.; XAVIER, J. A. D.; Engenharia de Reservatórios de Petróleo, Primeira Edição, Rio de Janeiro, Editora Interciência, 56 p., 2011.

SANTOS, F. K. G. et al. Seleção de tensoativos não iônicos para uso na recuperação avançada de petróleo. In: PDPETRO, 4, 2007, Campinas, Sp. 4PDPETRO_2_1_0067-2. Campinas, Sp: Copyright @ 2007 Abpg, 2007.

SANTOS, M. J. Estudo avançado para caracterização química de petróleo do campo de Carmópolis, Sergipe. 2013. Dissertação de Mestrado, Programa de Pós-Graduação em Química do Departamento de Química da Universidade Federal de Sergipe, 2013.

SCHRAMM, L. L., Emulsions: Fundamentals and Applications in the Petroleum Industry, Adv. Chem. Ser. Monograph Series 231, Am. Chem. Soc., Washington DC, 1992.

SOUZA, D. R. Q. A; OLIVEIRA, L. A.; FERREIRA, G. F. D.; LOBATO, A. K. C. L; SANTOS, L. C. L. Avaliação de Tensoativo Comerciais em Sistemas Microemulsionados na Quebra de Emulsão de Petróleo. In: I Congresso Nacional de Engenharia de Petróleo, Gás Natural e Biocombustíveis. Campina Grande, 2015.

SZKLO, A. Fundamentos de Refino do petróleo. $2^{\mathrm{a}}$ Ed. Interciência, Rio de Janeiro, 2005. 